\title{
Live Demonstration: Smart tracker and gesture capturer for people with Parkinson's diseases
}

\author{
José Ilton de Oliveira Filho ${ }^{1}$, Mariane Bianca de Melo Bezerra ${ }^{1,2}$, and Khaled Nabil Salama ${ }^{1}$ \\ ${ }^{1}$ King Abdullah University of Science and Technology (KAUST), Thuwal, Makkah Province, Kingdom of Saudi Arabia, \\ ${ }^{2}$ Federal University of Campina Grande (UFCG), Campina Grande, Paraiba State, Brazil, \\ E-mail: \{jose.deoliveirafilho, marieanne.demelobezerra, khaled.salama\}@kaust.edu.sa
}

\section{EARLIER PUBLICATION AND INTRODUCTION}

Parkinson's disease is classified as a chronic movement disorder whose incidence is proportional to the age. It is a common progressive neurodegenerative condition associated with significant disability and negative impact on the quality of life. Its manifestations involve difficulty with coordinated movements such as asymmetric resting tremor, rigidity, and bradykinesia. These symptoms and their response to levodopa constitute the basis for a clinical diagnosis of Parkinson's disease (PD) [1]. A recent study of this disease in North America showed the prevalence of PD among those aged $\geqslant 45$ years to be 572 per 100,000 , with 680,000 individuals in the US aged $\geqslant$ 45 years with $\mathrm{PD}$ in 2010 and the estimative to rise to approximately 930,000 in 2020 and 1,238,000 in 2030 based on the US Census Bureau's populational projections [2].

Equally important to its physical symptoms, people with PD commonly develop deep depression due to poorer quality of life, increased disability, worse outcomes, and greater caregiver burden [3]. To improve their quality of life, we propose a device that can help them navigate on internet and also support in small tasks in daily life such as open the doors. The simple fact of giving back dignity and independence to these people so that they are able to execute the simplest daily activities would improve their quality of life and help them overcome the burden given by the disease [4].

Allied to the improvement of the quality of life, the device will also monitor and continuously quantify the PD tremor potentially yielding to the optimization of uniquely individualized drug therapy strategies for the patient.

\section{DEMONSTRATION SETUP}

This live demonstration presents a bracelet sensor that involves the wrist of a person with PD. The bracelet acquires the data of the person's movements and interprets it matching with recorded gestures. Each change is stored and sent through Bluetooth (BLE) to the Main Controller. If this movement matches with saved gestures, it will execute commands such as open YouTube or Netflix, browse back or forward on YouTube or Netflix, select and play video. It will have the capability also to open a small door, with NFC protocol. Figure 1 shows the demonstration setup, where you can see the user manual, both wrist sensors, an additional sensor, the main controller, a raspberry pi, and the small door. Real-time graphs will be shown on a laptop and a smartphone through remote access software.
Although a computer and a smartphone will be used in the demonstration, they are not part of the setup.

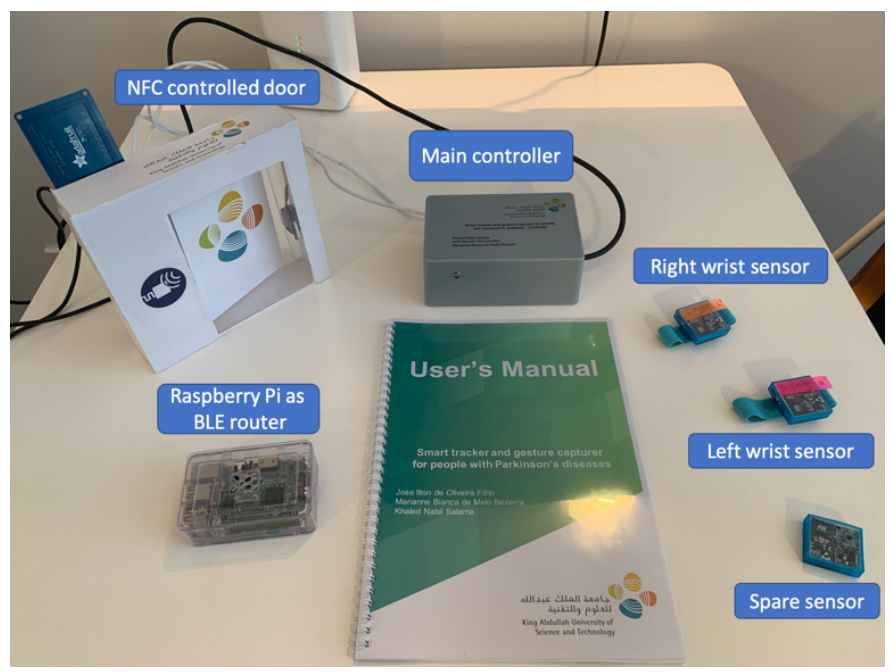

Fig. 1. Demonstration setup of the devices.

\section{VISITOR EXPERIENCE}

The visitors will be invited to immerse themselves in a fully interactive exhibit and test both functions of the device: gesture identifier and tracker. Once with the sensors, they will see the real-time plot of their movements, and by using the manual, they can try to execute any commands, for example, browser in the YouTube and play a video. The visitors will also be encouraged to simulate shriving and random shaking to mimic a real person with PD.

\section{REFERENCES}

[1] M. Lew, "Overview of Parkinson's Disease," Pharmacotherapy, vol. 27, no. 12 Part 2, p. 155S-160S, Dec. 2007. URL: https://doi.org/10.1592/phco.27.12part2.155S

[2] C. Marras et al., "Prevalence of Parkinson's disease across North America," npj Parkinson's Disease, vol. 4, no. 1, Jul. 2018. URL: https://doi.org/10.1038/s41531-018-0058-0

[3] A. Frenklach, "Management of Depression in Parkinson's Disease," American Journal of Psychiatry Residents' Journal, vol. 11, no. 4, pp. 811, Apr. 2016. URL: https://doi.org/10.1176/appi.ajp-rj.2016.110405

[4] Parkinson's Foundation, "Assistive Devices \& Home Health." URL: https://www.parkinson.org/sites/default/files/Assistive\%20Devices.pdf 